\title{
An Examination of Depression Self-Stigma in Asian and Caucasian Canadians
}

\author{
Alainna Wen ${ }^{1 *}$ and Andrew Szeto ${ }^{2}$
}

Depression is a serious mental disorder that is highly prevalent among Canadians. Stigma associated with depression is a key barrier in preventing depressed individuals from seeking psychological services. This type of help-seeking behavior is especially low in Asian individuals, which can be accounted for by the stigmatization of depression in this population. However, little research has been conducted on depression stigma in East Asian Canadians; specifically, those who are Chinese, Korean, and Japanese. Furthermore, no research has directly investigated self-stigma for depression in this population. The present study hypothesized that Asian Canadians would exhibit higher self-stigma for depression than Caucasian Canadians. To assess for this hypothesis, the present study recruited Asian and Caucasian undergraduate students and employed several surveys assessing depression stigma. Consistent with previous literature, the analyses revealed that the Asian participants had higher scores on some measures of social stigma, including attributions of blame, fear, and dangerousness towards a depressed individual. The present study indicated that Asian participants exhibited higher self-stigma, including an overall lower level of self-respect. However, there were no differences on any other stigma inventories. Possible explanations, limitations and future directions are discussed.

\section{INTRODUCTION}

Major Depressive Disorder (MDD) is defined by the Diagnostic and Statistical Manual (DSM-5) of Mental Disorders as having symptoms of depressed mood, diminished interest or pleasure in activities, significant changes in weight, sleep and motor activities, loss of energy, feelings of worthlessness, recurrent thoughts of death, and diminished cognitive abilities (American Psychiatric Association, 2000). MDD is highly prevalent in North America, and is associated with high rates of recurrence and non-recovery. A recent epidemiological study conducted in Canada suggests that the lifetime prevalence of a major depressive episode was $12.2 \%$ (Patten et al., 2006).

Utilization of Mental Health Services by Asians in North America

In Canada, the East Asian population - including those who are Chinese, Japanese, and Korean Canadian - accounts for more than $5 \%$ of the Canadian population and almost $30 \%$ of the visible minority population (Statistics Canada, 2006). Studies have shown that Asian individuals experience heightened depressive

\footnotetext{
${ }^{1}$ Department of Psychology, University of Notre Dame, Notre Dame, IN 46556
}

\footnotetext{
${ }^{2}$ Department of Psychology, University of Calgary, 539 Campus Place N.W., Calgary, AB T2N 1N4, Canada
}

\footnotetext{
*To whom correspondence should be addressed: awen@nd.edu

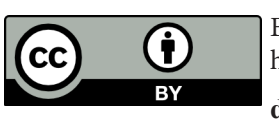

Except where otherwise noted, this work is licensed under https://creativecommons.org/licenses/by/4.0 doi:10.22186/jyi.34.5.17-25
}

symptoms when compared to Caucasians and other ethnic groups, and that suicide due to depression is the second leading cause of death for Asians residing in North America (National Alliance on Mental Illness, 2011). Despite this, Asian individuals residing in North America have been shown to underutilize almost every form of mental health services, and are significantly less like to seek help for mental health-related problems, compared to Caucasians (Atkinson \& Gim, 1989; Brown, 1998; Taylor et al., 2004). For example, a study by Le Meyer, Zane, Cho, and Takeuchi (2009) found that only $28 \%$ of Asian Americans use specialized mental health services compared to $54 \%$ of the general population. Furthermore, Asian Americans are significantly less likely to report psychological issues compared to somatic issues when seeking treatment (Yeung \& Kam, 2005). Finally, in cases where treatment is sought for mental health related issues, the dropout rate for Asian individuals is much greater than Caucasian individuals (Leong \& Lau, 2001). For instance, Sue (1977) found that 52\% of Asian Americans who sought help for mental health services dropped out after only one session, compared to $30 \%$ for Caucasian Americans. These results show that Asian individuals underutilize mental health services despite the prevalence and the debilitating outcomes of depression in this population (Yang \& Wongpat-Borja, 2007).

This disparity in rates of mental health service utilization for depression between Asians and Caucasians in North America has been proposed to be attributable to multiple causes, including cultural variations in symptom expression and attribution, practical barriers, and most importantly, social factors affecting the experience and disclosure of depression, such as stigma (Sue, Cheng, Saad, \& Chu, 2012). 


\section{Levels of Stigma: Social Stigma and Self-Stigma}

Stigma is defined as the co-occurrence of labeling, stereotyping, separation, status loss and discrimination in a situation where power is exercised (Link \& Phelan, 2001) and can be understood at different levels - namely, social stigma and self-stigma (see review by Ahmendani, 2011). Social stigma is the stigma held by a large fraction of society, where individuals with the stigmatized condition are deemed as less equal. This stigma can be internalized by an individual with the condition, resulting in self-stigma, which can cause that individual to feel guilty and inadequate about his or her condition (Corrigan, 2004).

Stigma related to mental illness, such as depression, is a significant public health issue as it presents a major barrier to help seeking and treatment-participation (Corrigan, 2004; Golberstein, Eisenberg, \& Gollust, 2008; Ting \& Hwang, 2009). Self-stigma can have deleterious effects on an individual's self-esteem and self-efficacy, which can lead to maladaptive coping mechanisms (Crocker \& Major, 1989). Previous studies have found that the expectation of being stigmatized, as well as the formation of selfstigma due to internalized public stigma, can have profound influence on psychological well being (Corrigan, Watson, \& Barr, 2006; Link, Cullen, Struening, Shrout, \& Dohrenwend, 1989). A recent study found that self-stigma mediated the relationship between depressive symptoms and professional help-seeking intentions (Lienemann, Siegel \& Crano, 2013). Taken together, the literature on self-stigma of mental illness suggests that internalized stigma prevents affected individuals from seeking treatment, which can result in poor mental health outcomes and death by suicide.

"Face" and the Stigma of Mental Illness in Asian Individuals Most of the research on depression stigma among Asians residing in North America has been conducted on Asian Americans. Relevant studies have shown that stigma is one of the primary reasons why many Asian Americans defer seeking treatment for depression (Leong \& Lau, 2001). Asian Americans indicate they experience greater depression stigma from Asian American friends, employers, and family compared to Caucasian Americans (Fogel \& Ford, 2005; Georg Hsu et al., 2008). This is important as the likelihood of an individual recognizing their need for seeking mental health services is negatively associated with perceived stigma, rendering it more challenging for said individuals to obtain help (Golberstein, Eisenberg, \& Gollust, 2008). As a result, many researchers have begun to examine the factors correlated with the stigma associated with depression, particularly in these Asian American populations.

Kleinman and Kleinman (1993) proposed that this stigma towards mental illness in Chinese individuals may be related to the loss of "face," or social reputation. Various studies examining the stigma of mental illness experienced by Chinese individuals in the context of family, community, and personal belief have confirmed this theory (Lam et al., 2010, Leong \& Lau, 2001; Lin \& Lin, 1981). Furthermore, Asian individuals residing in Western nations, compared to European counterparts, tend to explain depressive symptoms as social and moral problems (Karasz, 2005;
Karasz, Dempsey, \& Fallek, 2007; Xu et al., 2013, Yang et al., 2007). These findings were also present in examinations of the Japanese culture, as their culture greatly values group approval - especially from the family - and mental illness is viewed as a source of shame (Samuma, 1978). This can lead to family interfering with an individual's help seeking and treatment, resulting in poor mental health outcomes.

Studies that examined factors that influence depression stigma in the Asian American population focuses on cultural differences in values, beliefs, manifestation of symptoms, and treatment preferences. The emphasis on saving "face" or upholding one's social reputation and the negative attributes associated with mental illness (i.e., weakness of character, a punishment for one's transgressions in life) in Asian cultures has been proposed to result in experiences of heightened stigma (Gary, 2005; Lee, 1996; Pearson \& Yiu, 1993).

Self-Stigma of Depression and its Effects in Asian Individuals Despite the significant positive implications of identifying the effects of depression self-stigma on Asian individuals in North America, only a few studies have directly examined this line of research. A study by Lam et al. (2010) proposed that self-stigma is greater in Chinese individuals with mental illnesses compared to individuals residing in Western nations. Lam and colleagues suggest this intensified self-stigma is the result of an interaction between strong familial stigma and an increased likelihood of social stigma due to the cultural values of social harmony, familial orientation, and accepting one's role (Kirmayer, 1989). A recent study by Pedersen and Paves (2014) found a significant correlation between Asian ethnicity and self-stigma for mental health treatment; specifically, the results reported that Asian participants were more likely to view an individual negatively if they sought mental health treatment. The same study also found a significant association between greater self-stigma for mental illness and lower likelihood to seek treatment for mental disorders.

Gaps in the Literature on Depression Self-Stigma in Asian Canadians

When evaluated collectively, the limited literature available on the self-stigma of depression in these populations suggests that Asian individuals residing in North American exhibit greater internalized stigma for mental illness and their treatment. This self-stigma may account for the delay in seeking professional help and receiving efficient treatment, which in turn may result in prolonged depressive episodes and poor mental health outcomes. However, no study has examined self-stigma in the context of depression specifically in this population. Furthermore, a majority of the research on cultural differences in depression stigma between Asians and Caucasians have been conducted in the US, which is problematic given the variations in the health care systems and cultures of Canada and the US, and therefore limits the ability to extrapolate from these studies to Asian Canadians.

\section{The Present Study}

The growth of the Asian population in Canada underscores the 
need for studies assessing culture-specific beliefs and attitudes about depression to provide the basis for developing competent mental health services. Given the lack of literature on the experience of self-stigma associated with depression in Asian Canadians specifically, the purpose of this study was exploratory in nature. Nonetheless, there are clear patterns of results that mirror those from the Asian American stigma literature. In line with this previous literature, the current study predicted that Asian Canadians would report higher levels of social stigma towards depression, its treatment, and help seeking behaviours. As results from recent studies indicate that Asian Canadians may be more likely to internalize social stigma for mental illness, the present study further predicted that Asian Canadians would exhibit higher self-stigma associated with depression compared to Caucasian Canadians.

\section{METHODS}

\section{Participants}

The study sample consisted of 70 participants in two participant groups: Asian-Canadians $(n=31)$ and Caucasian-Canadians $(n$ =39). The participants were undergraduates at the University of Calgary and were recruited through the Department of Psychology's research participation pool. The study sample consisted of 56 women and 13 men, with the mean age of 21.39 years $(\mathrm{SD}=$ 7.15). Participants were compensated with 1 course credit for their involvement. Each participant met the inclusion criteria for either the Asian or the Caucasian Canadian group by identifying as either East Asian Canadian (e.g. Chinese, Japanese) or Caucasian Canadian (e.g. French, German).

\section{Dependent Measures}

Attribution Questionnaire (AW27; Corrigan et al., 2003). Participants completed the AQ27, a 27-item questionnaire that assesses how individuals view mental illness. This questionnaire has been modified to fit the context of depression by changing the words "mental illness" to "depression." The modified AQ27 consists of a vignette about Harry, an individual with depression, and uses a 7 -point Likert scale $(1=$ strongly disagree, $7=$ strongly agree) to assess various aspects of attributions, including blame, anger, pity, help, dangerousness, fear, avoidance, segregation, and coercion. Higher scores indicate greater stigmatizing attitudes and beliefs towards depression for all attribution except the Help subscale. The AQ27 has been evaluated as reliable and valid (Brown, 2008; Pinto, Hickman, Logsdon \& Burant, 2012). The current study found AQ27 to have acceptable internal consistency $(\alpha=$ $.75)$.

Endorsed and Anticipated Stigma Inventory (EASI; Vogt et al., 2014). Participants completed the EASI, a 40-item questionnaire that assesses stigma associated with mental illness. This questionnaire has been modified to fit the context of depression by changing the words "mental illness" to "depression." The modified EASI uses a 5-point Likert Scale ( $1=$ strongly disagree, $5=$ strongly agree) to assess beliefs about depression, its treatment and how to seek it, as well as concerns about stigma in the workplace and from loved ones. Higher scores indicate greater stigmatizing attitudes and beliefs towards depression. Preliminary assessments indicate that EASI has good reliability and validity (Vogt et al., 2014). The current study found EASI to have good internal consistency $(\alpha=.93)$.

Self-Stigma for Mental Illness Scale (SSMIS; Corrigan, Watson \& Barr, 2006). Participants completed the SSMIS, a 40item scale assessing self-stigma associated with mental illness. This questionnaire has been modified to fit the context of depression by changing the words "mental illness" to "depression." The modified SSMIS uses a 9-point Likert Scale ( 1 = strongly disagree, $9=$ strongly agree) to assess for various components of self-stigma, including the awareness, agreement and application of the stigma, as well as lower respect for self. Higher scores indicate greater self-stigma. The SSMIS has been shown to have good validity and reliability (Corrigan et al., 2012). The current study found the SSMIS to have good internal consistency $(\alpha=.95)$.

\section{Covariate Measures}

General Ethnicity Questionnaire (GEQ; Tsai, Ying \& Lee, 2000). Participants completed the GEQ, a 59-item questionnaire that assesses cultural background and identity and acculturation to Canadian culture. The GEQ uses a 5-point Likert Scale $(1=$ strongly disagree, $5=$ strongly agree). Good psychometric properties of the GEQ have been well documented (Huynh, Howell \& Benet-Martinez, 2009; Tsai, Ying \& Lee, 2000). The current study found the GEQ to have good internal consistency $(\alpha=.83)$.

Back Depression Inventory-II (BDI-II; Beck, Steer \& Brown). Participants completed the BDI-II, a 21-item questionnaire that assesses the severity of depressive symptoms over the past 2 weeks. Items are rated on a 4-point scale, where higher scores indicate greater depressive symptomatology. The strong psychometric properties of the BDI-II have been well documented in the general population (Beck et al., 1996; Dozois, Dobson, \& Ahnberg, 1998) as well as in the university population (Carmody, $2005)$. The current study found the BDI to have good internal consistency $(\alpha=.94)$.

Level of Contact-Report (LCR; Holmes et al., 1999). Participants completed the LCR, a 15-item questionnaire assessing frequency of exposure to and interaction with individuals with mental illness. This questionnaire has been modified to fit the context of depression by changing the words "mental illness" to "depression." The modified LCR consists of yes or no responses to 14 items, where higher scores indicate greater contact with depression. The LCR has been shown to have good reliability and validity (Corrigan et al., 2001). The current study found that the LCR did not meet conventional levels for acceptable internal consistency $(\alpha=.64)$.

\section{Other Measures}

Demographic Survey. Participants completed a demographics survey that asked about age, gender, level of education, and number and types of psychology courses taken.

Open-Ended Questionnaire (OEQ). Participants completed 
3 open-ended questions assessing knowledge of and experience with depression and its stigma. The questions were as follows: 1) In general, describe your knowledge of depression and how you acquired this knowledge; 2) In general, describe your knowledge of mental illness and how you acquired this knowledge; and 3) Describe your past involvement in research studies or programs related to the stigma of mental illness and when these involvements took place. Results for this questionnaire were not used in the study.

\section{PROCEDURE}

Participants for this study were recruited through the research participant pool, where they signed up to participate in either the Perceptions of Mental Illness in Asian Canadians study or the Perceptions of Mental Illness in Caucasian Canadians study at a specific timeslot. Both studies took place separately in a computer laboratory setting. Upon arrival, participants provided informed consent and then were given the link to the online study administered with Qualtrics@. All participants completed the questionnaires in the following order: demographics, GEQ, AQ27, EASI, SSMIS, BDIII, LCR, OEQ. The questionnaires were administered in this particular order for several reasons: first, cultural identity was measured before stigma to prime participants to think in a congruent fashion with their cultural identity when answering stigma questions; second, depressive symptoms were assessed after cultural identity and stigma to prevent any potential negative mood induction effects; and finally, the LCR and OEQ were placed at the end of the session to alleviate any negative emotions that may have resulted from completing the BDI. Upon completion, participants were fully debriefed.

\section{RESULTS}

\section{Preliminary Analyses}

There were no significant differences across Asian and Caucasian Canadians based on age, $t(68)=0.38, p=.159$, and sex, $\chi^{2}(2)=$ $0.271, p=.603$. As a result, age and sex were not included as covariates in the analyses of group differences in the stigma measures. The groups differed significantly on acculturation, as measured by the GEQ, $t(68)=-5.36, p<.001, d=-1.28$, where Asian participants had a lower level of acculturation than Caucasian participants. Hence, acculturation was included as a covariate in subsequent analyses where applicable.

Correlations between all variables in the whole sample and in the Asian and Caucasian samples are shown in Table 1. Previous literature on depression stigma suggested that contact with individuals with mental illness is negatively correlated with stigma (Corrigan et al., 2002). Therefore, it may be possible that individuals' contact with those who have depression is associated with their stigma towards depression. Indeed, the results showed significant correlations between LCR and AQ, $r=.462, p<.001$, EASI, $r=$ $.356, p=.003$, and SSMIS, $r=0.265, p=.033$. As a result, LCR scores were included as a covariate in the analyses of group differences in measures of AQ, EASI, and SSMIS. The BDI did not

\begin{tabular}{ccccccc}
\hline & AQ & EASI & SSMIS & BDI & GEQ & LCR \\
\hline AQ & .751 & -- & -- & -- & -- & -- \\
EASI & $.550^{* *}$ & .932 & -- & -- & -- & -- \\
SSMIS & $.326^{* *}$ & $.513^{* *}$ & .946 & -- & -- & -- \\
BDI & -.035 & .184 & .171 & .935 & -- & -- \\
GEQ & -.144 & -.024 & -.114 & -.001 & .829 & -- \\
\hline LCR & $.462 * *$ & $.346^{* *}$ & $.255^{*}$ & -.150 & -.069 & .635 \\
\hline
\end{tabular}

Table 1. Correlations between All Variables and Cronbach's Alpha for All Measures for the Whole Sample $(\boldsymbol{n}=\mathbf{7 0})$. AQ = Attribution Questionnaire. EASI $=$ Endorsed and Anticipated Stigma Inventory. SSMIS = Self Stigma for Mental Illness Scale. BDI = Beck Depression Inventory. GEQ = General Ethnicity Questionnaire. LCR $=$ Level of Contact Report. Cronbach's alphas are shown in the diagonal. $* p<.05$. $* * p<.01$.

correlate with any of the dependent measures and therefore is not included as a covariate in subsequent analyses.

Primary Analyses

A series of independent samples t-tests or analyses of covariance (ANCOVA) were conducted to examine the differences between Asian and Caucasian Canadians on the various dependent measures.

Hypothesis 1: Social stigma of depression in Asian and Caucasian Canadians. Means and standard deviations of scores on the Endorsed and Anticipated Stigma Inventory (EASI) and the Attribution Questionnaire (AQ) are shown in Table 2. ANCOVAs were conducted on the EASI controlling for GEQ and LCR to determine if Asian Canadians endorse greater stigmatizing beliefs and attitudes towards depression, its treatment and help-seeking as well as anticipate greater stigma from loved ones and the workplace compared to Caucasian Canadians. The analyses showed no significant effect of group on total EASI scores, $F(1,67)=0.11$, $p=.742, \eta_{\mathrm{p}}{ }^{2}=0.002$. No significant effects were observed across groups on any of the subscale scores, $p>.20$.

ANCOVAs were also conducted on the AQ27 scores controlling for GEQ and LCR to determine if Asian Canadians would attribute greater stigmatizing attitudes and beliefs towards Harry, an individual described to have depression in the vignette, compared to Caucasian Canadians. Results showed no significant effect of group on the total AQ27 scores, $F(1,67)=1.84, p=.179, \eta_{\mathrm{p}}{ }^{2}=$ 0.027 . Within the subscales, there was a non-significant trend of group on the Blame subscale scores, $F(1,67)=3.96, p=.051$, $\eta_{\mathrm{p}}{ }^{2}=0.056$, where Asian participants exhibited higher blame towards Harry compared to Caucasian participants. There was also a non-significant trend for Dangerousness subscale scores, $F(1,67)$ $=2.96, p=.090, \eta_{\mathrm{p}}{ }^{2}=0.042$, and Fear subscale scores, $F(1,67)$ $=2.98, p=.089, \eta_{\mathrm{p}}^{2}=0.043$, where Asian participants reported 


\begin{tabular}{|c|c|c|c|c|}
\hline & \multicolumn{2}{|c|}{$\underline{\text { Asian Canadians }}$} & \multicolumn{2}{|c|}{ Caucasian Canadians } \\
\hline & \multicolumn{2}{|c|}{$n=31$} & \multicolumn{2}{|c|}{$n=39$} \\
\hline & $M$ & $S E$ & $M$ & $S E$ \\
\hline EASI & 96.38 & 3.56 & 94.78 & 3.16 \\
\hline Beliefs about Depression & 17.08 & 0.72 & 15.96 & 0.64 \\
\hline Beliefs about Depression Treatment & 17.19 & 0.77 & 17.08 & 0.68 \\
\hline Beliefs about Seeking Treatment & 21.73 & 1.24 & 20.99 & 1.10 \\
\hline Concerns about Stigma from Loved Ones & 17.33 & 1.13 & 17.00 & 1.01 \\
\hline Concerns about Stigma in the Workplace & 23.06 & 1.22 & 23.75 & 1.08 \\
\hline$A Q 27$ & 114.89 & 30.94 & 113.45 & 26.92 \\
\hline Blame & 8.94 & 0.70 & 7.04 & 0.62 \\
\hline Anger & 5.96 & 0.74 & 6.85 & 0.63 \\
\hline Pity & 16.49 & 0.46 & 16.69 & 0.41 \\
\hline Help & 20.83 & 0.72 & 21.47 & 0.64 \\
\hline Dangerousness & 6.54 & 0.63 & 5.06 & 0.60 \\
\hline Fear & 5.86 & 0.57 & 4.53 & 0.50 \\
\hline Avoidance & 15.72 & 0.62 & 16.41 & 0.55 \\
\hline Segregation & 5.44 & 0.49 & 5.17 & 0.44 \\
\hline Coercion & 13.13 & 0.78 & 11.98 & 0.69 \\
\hline SSMIS & 150.21 & 8.33 & 120.41 & 7.39 \\
\hline Awareness & 50.65 & 3.30 & 47.29 & 2.93 \\
\hline Agreement & 25.95 & 1.60 & 18.47 & 1.42 \\
\hline Application & 33.05 & 3.12 & 26.48 & 2.77 \\
\hline Hurt Self & 40.56 & 3.24 & 28.18 & 2.88 \\
\hline
\end{tabular}

Table 2. Means and Standard Errors of AQ27, EASI, and SSMIS scores by group. $M=$ mean score. SE $=$ standard error. AQ $=$ Attribution Questionnaire. EASI = Endorsed and Anticipated Stigma Inventory. SSMIS = Self-Stigma for Mental Illness Scale. 
Harry as more dangerous and fear-inducing than Caucasian participants. There were no significant effects of group on the scores of the other subscales, $p>.10$.

Hypothesis 2: Self-Stigma associated with depression in Asian and Caucasian Canadians. ANCOVAs were conducted on the SSMIS and its subscales controlling for GEQ and LCR to determine if Asian Canadians exhibit higher self-stigma associated with depression than Caucasian Canadians. Means and standard deviations are shown in Table 2. The analyses revealed a significant effect of group on the total SSMIS scores, $F(1,67)=6.89$, $p=.011, \eta_{\mathrm{p}}{ }^{2}=.0 .093$, where Asian participants showed greater self-stigma than Caucasian participants. Within the subscales, there was a significant effect of group on scores on the Agreement subscale, $F(1,67)=11.82, p=.001, \eta_{\mathrm{p}}{ }^{2}=0.150$, and on the Hurt Self subscale, $F(1,67)=7.86, p=.007, \eta_{p}^{2}=0.105$, where Asian participants reported higher agreement to the social stigma of depression and indicated a lower level of respect to self if they had depression compared to Caucasian participants. There were no significant effects of group on scores on the other subscales.

\section{DISCUSSION}

Stigma is an important factor in preventing individuals with mental disorders such as depression from seeking help. Given that stigma is shaped by culture, the extant Caucasian-focused research literature cannot adequately explain or address depression stigma in Asian individuals. Furthermore, there exist research gaps in this literature as little research has focused on the Asian Canadian (as opposed to the Asian American) experience of depression stigma, and there is a paucity of research on self-stigma for depression. The current study examined internalized stigmatizing attitudes and beliefs towards depression in Asian and Caucasian-Canadian student samples to address some of these gaps.

\section{Evaluation of Hypotheses}

Hypothesis 1: Stigmatizing attitudes and beliefs towards depression in Asian and Caucasian Canadians. As predicted, Asian individuals showed higher blame towards Harry, the individual in the vignette, for his depression compared to their Caucasian counterparts. Additionally, there was a trend in which the Asian participants believed Harry was more fearful and dangerous as compared to the Caucasian participants. These findings are consistent with previous studies that found Asian Americans exhibited greater stigma towards those with depression as compared to European Americans (Cheng, 2014; Parcespepe \& Cabassa, 2014). However, there were no significant group differences on overall scores on the AQ as well as on the other subscales, namely, Anger, Pity, Help, Avoidance, Segregation, and Coercion.

Contrary to the hypothesis, no significant differences were found between Asian and Caucasians on endorsed stigma regarding depression etiology, its treatment and help-seeking, as well as anticipated depression stigma from loved ones or others workplace, as measured by the EASI. These null results are inconsistent with findings in the literature that Asian individuals experience greater depression stigma from friends, employers, and family compared to Caucasian Americans (Fogel \& Ford, 2005, Lam et al., 2012). The null results on the EASI and on some AQ27 scores may be accounted for by the fact that a majority of the study sample consisted of university students who were taking at least one psychology course. The current literature suggests that mental health knowledge has been found to moderate attitudes towards mental illnesses (Angermeyer \& Dietrich). This is supported by the fact that both Asian and Caucasian participants scored low on various stigma inventories. The education received by the college students may have masked any group differences associated with cultural background. Findings on other subscales, however, indicate that Asian Canadians exhibit higher levels of stigma towards depression (i.e., in respect to blame, fear, and perception of dangerousness) and add support to existing findings in the literature on higher depression stigmatization in Asian individuals (Cheng, 2014; Yang \& Wonpat-Borja, 2007). It is unclear why the sample characteristics would mask some group differences but not others; future studies are needed to account for other factors that can explain these findings.

Hypothesis 2: Self-stigma associated with depression in Asian and Caucasian Canadians. As predicted, Asian participants scored significantly higher for self-stigma as measured by the SSMIS when compared to Caucasian participants. Specifically, Asian individuals indicated greater agreement with the social stigma associated with depression (Agreement subscale) and a lower respect for themselves if they were experiencing depression (SelfHurt subscale). This finding is consistent with research literature that suggested a higher pressure to conform to the society and to adhere to social roles in Asian cultures compared to Western cultures could increase the internalization of social stigma in Asian individuals and result in higher self-stigma (Kirmayer, 1989; Ng, 1997). This finding is also consistent with findings by Pedersen \& Paves (2014) that indicated a significant association between Asian ethnicity and self-stigma for mental health treatment.

However, no group differences were found for the degree to which participants were aware of the stigma associated with depression (Awareness subscale) and the degree to which they apply these stigmatizing attitudes and beliefs to themselves (Application subscale). The null results in these self-stigma measures, which were like those in social stigma measures, may be due to the exposure to information about depression in participants' psychology education. In addition, these findings may be explained by the fact that participants in the present study did not have a clinical sample; it is reasonable to assume that the absence of depressive symptoms and diagnosis may have limited the experience of perceiving stigma associated with depression and internalizing this as self-stigma. A study by Hoge et al. (2004), for example, found that individuals who met criteria for a mental illness were twice as likely as others to report concern about possible stigmatization and other barriers to seeking mental health care. Nonetheless, significant results on the overall measure of stigma and the specific measures of Agreement and Self-Hurt suggests that Asian participants experience higher self-stigma for depression than Caucasian 
participants.

\section{Implications}

The present study found some significant differences in self-stigma associated with depression using various measures. The study suggests that Asian Canadians may experience greater self-stigma (i.e., greater agreement with social stigma and lower respect for self) compared to Caucasian Canadians. Addressing self-stigma among Asian Canadians is important, as internalized stigma can lead to a lower of seeking professional help independently of social stigma. The findings in the present study suggests that it is important for clinicians to address this in practice with Asian $\mathrm{Ca}$ nadians to improve mental health outcomes in this population. Clinicians may choose to speak about the prevalence of depression as well as the positive outcomes of treatment to reduce the feelings of inferiority in Asian patients with depression, but should be aware that Asian individuals may be less likely to talk openly about their depressive symptoms and may be more likely to view themselves negatively because of their mental illness. Thus, clinicians should pay closer attention to any indicators that patients are suffering from depression and to provide adequate information and support to Asian Canadian patients to increase their help-seeking behaviors, treatment adherence, and good mental health outcomes. Antistigma interventions in the community (i.e., flyers, seminars) that focuses on disseminating information regarding the importance of and misconceptions related to mental health can work towards reducing stigma and improving treatment-seeking behaviors. However, such implications would be strengthened if results from this study are replicated with a greater sample size and appropriate statistical corrections.

Research on cultural differences in the stigmatization of depression has two general aims: one, to understand the similarity and differences in the experience of stigma; and two, to assess for methods to alleviate this stigma in a culturally competent fashion. Corrigan and Penn (1999) identified three approaches towards reducing social stigma towards mental illness, including protest, education, and contact. However, the relevancy and success of these de-stigmatization strategies have yet been shown in the Asian Canadian population. Given research that suggested a greater emphasis on social reputation (Kleinman \& Kleinman, 1993) and a greater agreement with social stigma (Lam et al., 2010) in Asian individuals, effective methods for reducing stigma may look very different for this group compared to those for the general population. For instance, interventions may benefit from focusing more on reducing patient self-blame, as Asian individuals may exhibit greater blame towards individuals with depression as well as harbor greater self-stigma. In addition, given that Asian Canadians may experience greater self-stigma than Caucasian Canadians, effective interventions to reduce self-stigma specifically should be studied to reduce this internalized depression stigma in Asian Canadians. Although the present study offers preliminary evidence for the differential experience of the stigmatization of depression in Asian and Caucasian Canadians, research on culturally competent interventions to reduce self-stigmatization in Asian Canadians is needed once the present study's results are confirmed.

\section{Limitations and Future Directions}

The present study has several limitations. First, because this study was exploratory in nature and was based on correlational analyses, the results do not provide strong inferences for causal relations and should be viewed as preliminary. The results of this research should be replicated and cross-validated in future studies that test specific hypotheses apriority and have planned comparisons. Additionally, the present study had a relatively small sample size, which cast limitations on the power to find effects. Future studies with adequate sample sizes obtained from power analysis are needed to confirm the results of the present study. Furthermore, participants were recruited from an undergraduate psychology student sample. As previously explained, this limits the generalizability of the present study's findings. Although the sample consisted of youths, who are more susceptible to affective disorders due to their age group (Dobson \& Dozois, 2011), future studies should utilize a community sample to assess for stigmatizing attitudes and beliefs towards depression in Asian Canadians to increase generalizability to all age groups. The study sample was also not limited to clinically depressed participants. A sample of clinically depressed participants would have been more adequate for examining self-stigma. Although analyses showed no significant correlation between depressive symptoms and self-stigma, the lack of experience with depression could significantly reduce the validity of self-reports on levels of self-stigmatization of depression. Future studies should pre-screen participants for depression to conduct a more valid assessment of self-stigma in individuals who are clinically depressed.

With regards to study design, a key limitation lies in the fact that although the present study measured cultural identity and acculturation to Canadian culture, the extent to which Asian participants adhered to cultures other than that of Canada (China, Japan, Korea) was not measured. This is important because acculturation for Asian participants in this study would have been more accurately measured by their adoption of Canadian beliefs, values, and practices as well as the maintenance of their heritage culture (Chinese beliefs, values, and practices) (see Berry, 2005); however, only the adoption of Canadian beliefs was measured in this study. In addition, a majority of the Asian Canadian participant group was also born in Canada. To accurately assess the effect of acculturation on depression stigma, future studies should utilize an immigrant sample and measure place of birth, length of stay in Canada and participant acculturations to Canadian culture and to their heritage culture. Furthermore, although the study aimed to assess depression stigma in Asian Canadians, it is important to acknowledge the potential differences in the stigmatizing attitudes and beliefs of depression in different Asian cultures (Chinese vs. Korean). It would be difficult to assess differences among these cultures in the present study due to the relatively small sample. Future studies should compare the stigmatization of depression in various Asian cultures by recruiting individuals who identify with a specific Asian culture and administer measures of acculturation 
and cultural identification to these groups separately. This method allows for a greater comprehensive understanding of the influence of specific cultures on stigma of depression, which in turn would facilitate efforts to improve help-seeking behaviors in these populations as well as cultural competency in clinical practice in Canada.

\section{CONCLUSION}

With the increasing diversity of the Canadian population, importance of cross-cultural knowledge, and application of depression research, the assessment of cultural variations in the stigmatization of depression is becoming increasingly important. This study offers preliminary findings of differences in self-stigmatizing attitudes and beliefs towards depression in Asian and Caucasian Canadians. Compared to Caucasian Canadians, Asian Canadians exhibited higher agreement to the social stigmatization of depression and demonstrated higher self-stigma associated with depression. An awareness of cultural differences and similarities is crucial towards building cultural competence in mental health services and to improve mental health outcomes in societies with diverse cultural groups such as Canada.

\section{REFERENCES}

Ahmedani, B. K. (2011). Mental health stigma: Society, individuals, and the profession. Journal of social work values and ethics, 8(2), 4-1.

American Psychiatric Association (2000). Diagnostic and statistical manual of mental disorders (4th ed., Text Revision). Washington, DC: Author.

Angermeyer, M. C., \& Dietrich, S. (2006) Public beliefs about and attitudes towards people with mental illness: a review of population studies. Acta Psychiatrica Scandinavica: 41, 163-179. doi:10.1111/j.1600-0447.2005.00699.x

Atkinson, D. R., \& Gim, R. H. (1989). Asian-American cultural identity and attitudes toward mental health services. Journal of Counseling Psychology, 36(2), 209. doi:10.1037/0022-0167.36.2.209

Beck, A. T., Steer, R. A., \& Brown, G. K. (1996). Beck Depression InventorySecond Edition. San Antonio, TX: Psychological Corporation.

Berry, J. W. (2005). Acculturation: Living successfully in two cultures. International journal of intercultural relations, 29(6), 697-712. doi:10.1016/j.ijintrel.2005.07.013

Brown, C. (1998). Underutilization of Mental Health Services by Asian-Americans residing in the United States. Issues in Mental Health Nursing, 19(3), 225240. doi: $10.1080 / 016128498249042$

Brown, S. A. (2008). Factors and measurement of mental illness stigma: A psychometric examination of the Attribution Questionnaire. Psychiatric rehabilitation journal, 32(2), 89. doi:10.2975/32.2.2008.89.94

Carmody, D. P. (2005). Psychometric characteristics of the Beck Depression Inventory-II with college students of diverse ethnicity. International Journal of Psychiatry in Clinical Practice, 9, 22-28. doi:10.1037/t00742-000

Cheng, Z. H. (2014). Asian Americans and European Americans' stigma levels in response to biological and social explanations of depression. Social Psychiatry and Psychiatric Epidemiology, 50(5), 767-776. doi:10.1007/s00127-0140999-5

Corrigan, P. W. (2004). How stigma interferes with mental health care. American Psychologist, 59, 614-625. doi:10.1037/0003-066X.59.7.614

Corrigan, P. W., Green, A., Lundin, R., Kubiak, M. A., \& Penn, D. L. (2001). Familiarity with and social distance from people who have serious mental illness. Psychiatric Services, 52, 953-958. doi:10.1176/appi.ps.52.7.953

Corrigan, P. W., \& Penn, D. L. (1999). Lessons from social psychology on discrediting psychiatric stigma. American Psychologist, 54(9), 765. doi:10.1037/0003-066X.54.9.765

Corrigan, P. W., Markowitz, F. E., Watson, A., Rowan, D., \& Kubiak, M. A. (2003). An attribution model of public discrimination towards persons with mental illness. Journal of Health and Social Behavior, 44(2), 162-79. doi: $10.2307 / 1519806$

Corrigan, P. W., Morris, S. B., Michaels, P. J., Rafacz, J. D., \& Rüsch, N. (2012). Challenging the public stigma of mental illness: a meta-analysis of outcome studies. Psychiatric Services, 63(10), 963-973. doi:10.1176/appi. ps.201100529

Corrigan, P. W., Rowan, D., Green, A., Lundin, R., River, P., Uphoff-Wasowski, K., White, K., \& Kubiak, M. A. (2002). Challenging two mental illness stigmas: Personal responsibility and dangerousness. Schizophrenia Bulletin, 28, 293309. doi:10.1037/0003-066X.54.9.765

Corrigan, P. W., Watson, A. C., \& Barr, L. (2006). The self-stigma of mental illness: Implications for self-esteem and self-efficacy. Journal of social and clinical psychology, 25(8), 875-884. doi:10.1521/jscp.2006.25.8.875

Crocker, J., \& Major, B. (1989). Social stigma and self-esteem: The self-protective properties of stigma. Psychological review, 96(4), 608. doi:10.1037/0033295X.96.4.608

Dobson, K. S., \& Dozois, D. J. (Eds.). (2011). Risk factors in depression. Academic Press.

Dozois, D. J. A, Dobson, K. S., \& Ahnberg, J. L. (1998). A psychometric evaluation of the Beck Depression Inventory - II. Psychological Assessment, 10(2), 83-89. doi:10.1037/1040-3590.10.2.83

Fogel, J., \& Ford, D. E. (2005). Stigma beliefs of Asian Americans with depression in an internet sample. Canadian Journal of Psychiatry, 50(8), 470.

Gary, F.A. (2005). Stigma: barrier to mental health care among ethnic minorities. Issues in Mental Health Nursing, 26(10), 979-999. doi:10.1080/01612840500280638

Georg Hsu, L. K., Wan, Y. M., Chang, H., Summergrad, P., Tsang, B. Y., \& Chen, H. (2008). Stigma of depression is more severe in Chinese Americans than Caucasian Americans. Psychiatry: Interpersonal and Biological Processes, 71(3), 210-218. doi:10.1521/psyc.2008.71.3.210

Golberstein, E., Eisenberg, D., \& Gollust, S. E. (2008). Perceived stigma and mental health care seeking. Psychiatric Services, 59, 392-399. doi:10.1176/appi. ps.59.4.392

Hoge, C. W., Castro, C. A., Messer, S. C., McGurk, D., Cotting, D. I., \& Koffman, R. L. (2004). Combat duty in Iraq and Afghanistan, mental health problems, and barriers to care. New England Journal of Medicine, 351(1), 13-22. doi:10.1056/NEJMoa040603

Holmes, E.P., Corrigan, P.W., \& Williams, P. Changing attitudes about schizophrenia. (1999). Schizophrenia Bulletin, 25, 447-56. doi:10.1093/oxfordjournals. schbul.a033392

Huynh, Q., Howell, R.T., \& Benet-Martinez, V. (2009). Reliability of Bidimensional Acculturation Scores: A Meta-Analysis. Journal of Cross-Cultural Psychology, 40, 256. doi:10.1177/0022022108328919

Karasz, A. (2005). Cultural differences in conceptual models of depression. Social Science and Medicine, 60, 1625-1635. doi:10.1016/j.socscimed.2004.08.011

Karasz, A., Dempsey, K., \& Fallek, R. (2007). Cultural differences in the experience of everyday symptoms: a comparative study of South Asian and European American women. Culture, Medicine, and Psychiatry, 31, 473-497. doi:10.1007/s11013-007-9066-y

Kirmayer, L. (1989) Cultural variations in the response to psychiatric disorder and emotional distress. Social Science and Medicine; 29, 327-329. doi:10.1016/0277-9536(89)90281-5

Kleinman, A., \& Kleinman, J. (1993). Face, favor and families: the social course of mental health problems in China and American societies, Chinese Journal of Mental Health, 6, 37- 47.

Lam, C. S., Tsang, H. W., Corrigan, P. W., Lee, Y. T., Angell, B., Shi, K., ... \& Larson, J. E. (2010). Chinese lay theory and mental illness stigma: Implications for research and practices. Journal of Rehabilitation, 76(1), 35.

Lee, E. (1996). Chinese families. In McGoldrick, J. Giordano, \& J.K. Pearce (Eds.), Ethnicity and family therapy (2nd., pp.248-267). New York: Guildford.

Leong, F. T., \& Lau, A. S. (2001). Barriers to providing effective mental health services to Asian Americans. Mental Health Services Resesarch, 3, 201-14. doi:10.1023/A:1013177014788

Le Meyer, O., Zane, N., Cho, Y., \& Takeuchi, D. T. (2009). Use of specialty mental health services by Asian Americans with psychiatric disorders. Journal of Consulting and Clinical Psychology, 77, 1000-1005. doi:10.1037/a0017065 
Lienemann, B. A., Siegel, J. T., \& Crano, W. D. (2013). Persuading people with depression to seek help: respect the boomerang. Health communication, 28(7), 718-728. doi:10.1037/t00524-000

Lin, T. L., \& Lin, M. C. (1981) Love, denial and rejection: responses of Chinese families to mental illness. In: Kleinman A, Lin T, eds. Normal and abnormal behaviour in Chinese culture. Dordrecht: Reidel, 387401.

Link, B. G., Cullen, F. T., Struening, E., Shrout, P. E., \& Dohrenwend, B. P. (1989). A modified labeling theory approach to mental disorders: An empirical assessment. American Sociological Review, 400-423. doi:10.2307/2095613

Link, B. G., \& Phelan, J. C. (2001) Conceptualizing stigma. Annual Review of Sociology, 27, 363-385. doi:10.1146/annurev.soc.27.1.363

National Alliance on Mental Illness (2011). Asian American and Pacific Islander (AA/PI) Community and Mental Health Fact Sheet.

Parcesepe, A. M., \& Cabassa, L. J. (2013). Public stigma of mental illness in the United States: a systematic literature review. Administration and Policy in Mental Health and Mental Health Services Research, 40(5), 384-399. doi:10.1007/s10488-012-0430-z

Patten, S. B., Wang, J.L., Williams, J. V. A., Currie, S., Beck, C. A., Maxwell, C. J., \& Guebaly, N. (2006). Descriptive Epidemiology of Major Depression in Canada. Canadian Journal of Psychiatry, 51, 84-90. doi:10.1037/t02121-000

Pearson, V., \& Yiu, M. K. (1993). Public attitudes towards mental health facilities: A study in Hong Kong. Social Work and Social Sciences Review, 4, 59-82.

Pedersen, E. R., \& Paves, A. P. (2014). Comparing perceived public stigma and personal stigma of mental health treatment seeking in a young adult sample. Psychiatry research, 219(1), 143-150. doi:10.1037/t01528-000

Pinto, M. D., Hickman, R., Logsdon, M. C., \& Burant, C. (2012). Psychometric evaluation of the revised attribution questionnaire (r-AQ) to measure mental illness stigma in adolescents. Journal of Nursing and Measurement, 20(1), 47-58. doi: $10.1037 / \mathrm{t} 23348-000$

Samuma, K. (1978) The Japanese family in relation to people's health. Social Science and Medicine; 12A, 419-427.

Statistics Canada. (2006). Census of Canada topic based tabulations, population estimates and projections: visible minority population table. Retrieved on April 2, 2015 from http://www.statcan.gc.ca/tables-tableaux/sum-som/101/cst01/ demo50a-eng.htm.

Sue, S., Cheng, J. K. Y., Saad, C. S., \& Chu, J. P. (2012). Asian American mental health: a call to action. American psychologist, 67(7), 532. doi:10.1037/ a0028900

Taylor, S. E., Sherman, D. K., Kim, H. S., Jarcho, J., Takagi, K., \& Dunagan, M. S., (2004). Culture and social support: who seeks it and why? Journal of Personality and Social Psychology, 87, 354-362. doi:10.1037/0022-3514.87.3.354

Ting, J. Y., \& Hwang, W. C. (2009). Cultural influences on help-seeking attitudes in Asian American students. American Journal of Orthopsychiatry, 79, 125 132. doi: $10.1037 / \mathrm{t} 05375-000$

Tsai, J. L., Ying, Y.-W., \& Lee, P. A. (2000). The meaning of "being Chinese" and "being American": Variation among Chinese American young adults. Journal of Cross-Cultural Psychology, 31, 302-331. doi:10.1177/0022022100031003002

Vogt, D., Di Leone, B. A. L., Sayer, N. A., Pineles, S. L., \& Litz, B. T. (2014). Endorsed and Anticipated Stigma Inventory (EASI): A Tool for Assessing Beliefs About Mental Illness and Mental Health Treatment Among Military Personnel and Veterans. Psychological Services, 11, 105-113. doi:10.1037/ t30266-000

Xu, M., Zou, L., Wilde, A., Meiser, B., Barlow-Stewart, K., Chan, B., ... \& Schofield, P. R. (2013). Exploring Culture-Specific Differences in Beliefs about Causes, Kinship and the Heritability of Major Depressive Disorder: The Views of Anglo-Celtic and Chinese-Australians. Journal of genetic counseling, 22(5), 613-624.

Yang, L. H., Kleinman, A., Link, B. G., Phelan, J.C., Lee, S., \& Good, B. (2007). Culture and stigma: adding moral experience to stigma theory. Soc. Sci. Med., 64(7), 1524-1535. doi:10.1016/j.socscimed.2006.11.013

Yang, L. H., \& Wonpat-Borja, A. (2007). Psychopathology among Asian-Ameri cans. In F. T. Leong, A. G. Inman, A. Ebreo, L. H. Yang, L. Kinoshita, \& M. Fu (Eds.), Handbook of Asian American psychology (2nd ed.). (pp. 379-405) Thousand Oaks, CA: Sage.

Yeung, A. S., \& Kam, R. (2005). Illness beliefs of depressed Asian Americans in primary care. In: Georgiopoulos, A.M., Rosenbaum, J.F. (Eds.), Perspectives in Cross-Cultural Psychiatry. Lippincott Williams \& Wilkins, Philadelphia, pp. 21-36. 